\title{
Educación socioemocional: discutiendo su implementación en el contexto educativo chileno ${ }^{1}$
}

\author{
Socio-Emotional Education: Discussing its Implementation Within \\ Chilean Educational Context
}

Pamela Barría-Herrera ${ }^{2}$, José Manuel Améstica-Abarca ${ }^{3}$; Christian Miranda-Jaña ${ }^{4}$

\begin{abstract}
RESUMEN
Las consecuencias de la crisis sanitaria, social y política reciente han planteado nuevos desafíos al sistema educativo chileno. Entre estos desafíos se encuentra propiciar el desarrollo socioemocional de sus estudiantes. En este contexto, distintos modelos y perspectivas sobre la educación y el aprendizaje socioemocional han adquirido gran relevancia y, a la vez, han puesto en evidencia que su implementación en el contexto educativo chileno requiere discutir aspectos como (i) la perspectiva a adoptar en torno a las emociones en los procesos educativos, (ii) la forma de comprender el rol de estudiantes y docentes involucrados/as en su implementación y (iii) el propio posicionamiento frente al desarrollo socioemocional como propuesta educativa. Estos elementos son discutidos en el presente ensayo.
\end{abstract}

Palabras claves: aprendizaje socioemocional; educación socioemocional; trabajo docente; rol docente; desarrollo de estudiantes.

\begin{abstract}
The consequences of recent health, social and political crisis have opened up new challenges for Chilean educational system, such as promoting social and emotional development among students. Within this framework, different models and perspectives regarding socio-emotional education and learning have gained relevance. Nevertheless, implementing these models in Chilean educational contexts requires discussing topics related to i) perspectives that may be adopted on emotions within educational processes, (ii) ways of understanding student's and

\footnotetext{
${ }^{1}$ Elaboración asociada a Proyecto FONDECYT 1181772.

${ }^{2}$ Estudiante de Doctorado en Educación, Universidad Metropolitana de Ciencias de la Educación (Santiago, Chile); magíster en Psicología Educacional, Universidad de Chile; pamela.barria@umce.cl.

${ }^{3}$ Coordinador de Vinculación con el Medio del proyecto Liderando Escuelas, Facultad de Educación, Universidad Diego Portales (Santiago, Chile); magíster en Psicología Educacional, Universidad de Chile; jose.amestica@udp.cl.

${ }^{4}$ Profesor Asociado, Departamento de Educación, Facultad de Ciencias Sociales, Universidad de Chile (Santiago, Chile); Doctor en Ciencias de la Educación, Pontificia Universidad Católica de Chile; christian.miranda@u.uchile.cl
} 
teacher's roles in its implementation, and (iii) our own position upon social and emotional learning as an educational proposal. These elements are discussed in this essay.

Keywords: Socio-emotional learning; socio-emotional education; teacher labour; teacher role; student development.

\section{Introducción}

Durante los últimos meses, los sistemas educativos han resentido los cambios sociales, políticos, económicos y ambientales asociados a la pandemia del COVID-19 y a las distintas medidas tomadas por gobiernos y autoridades, las cuales han buscado abordar las alteraciones que ha sufrido la vida cotidiana y sus efectos en las personas. Para responder a la incertidumbre, se han generado documentos y orientaciones que intentan promover el bienestar y la salud mental de la ciudadanía. Ejemplo de lo anterior, en el contexto chileno, han sido las orientaciones emitidas por los ministerios de Salud y Educación (Ministerio de Educación [Mineduc], 2020; Ministerio de Salud, 2020), así como informes emanados desde organismos técnicos donde participan distintas entidades de la sociedad civil (p. ej. Mesa Social COVID-19, 2020). De la misma manera, las orientaciones internacionales han indicado la necesidad de contar con iniciativas para apoyar los procesos de aprendizaje en este nuevo escenario (Organización para la Cooperación y el Desarrollo Económicos, 2020), aun cuando estas han debido acomodarse a entornos virtuales y, por lo tanto, a nuevas modalidades para mantener las relaciones humanas en el proceso educativo (Fondo de las Naciones Unidas para la Infancia [Unicef], 2020).

Particularmente, en el contexto de emergencia, el Ministerio de Educación de Chile (Mineduc, 2020) ha realizado diversas acciones que se han dirigido tanto a las familias, con recomendaciones para implementar en el hogar, como a directivos y docentes, promoviendo la construcción de vínculos dentro de la comunidad educativa, por medio de prácticas de educación remota. Además, desde el enfoque de la psicología positiva, se implementó la Bitácora para Docentes y un ciclo de webinars de aprendizaje socioemocional, en el que se abordaron temas como vinculación docente-estudiante, estrategias para fomentar el bienestar docente, estrategias para la contención y promoción de la resiliencia en tiempos de crisis, entre otros. En general, las acciones emanadas desde 
autoridades locales se han sumado a lo señalado por organismos internacionales y por las organizaciones de la sociedad civil.

En el contexto educacional, se ha puesto especial interés en reconocer la dimensión emocional dentro de los procesos de aprendizaje, lo que no es una inquietud nueva. En los últimos años, esta discusión se ha enfocado tanto en el trabajo docente (Cornejo, 2012; Weinstein, 2018) como en la experiencia estudiantil (Milicic et al., 2013; Agencia de Calidad de la Educación, 2018). A partir de ello, se puede afirmar que la preocupación por los procesos emocionales involucrados en la experiencia escolar ha sido relevada de manera sistemática durante los últimos años.

Para atender las circunstancias actuales, una de las propuestas que ha adquirido mayor fuerza es la de los modelos de educación o aprendizaje social y emocional (en inglés, social emotional learning, SEL, o social emotional education, SEE) (Bisquerra, 2000; Collaborative for Academic, Social and Emotional Learning [CASEL] ${ }^{5}$, 2017). Estos modelos han sido definidos de maneras similares, pero estableciendo ciertas distinciones. Por ejemplo, para Bisquerra (2000) la educación emocional sería un proceso que busca "potenciar el desarrollo emocional como complemento indispensable del desarrollo cognitivo" (p.243), rescatando su relevancia para la formación integral. Por otro lado, organizaciones como CASEL (2017) han traducido el aprendizaje socioemocional a un conjunto de dimensiones y competencias consideradas claves, las cuales incluyen: autocontrol, conciencia de sí mismo/a, autovaloración y autoconocimiento, autonomía y toma de decisiones, habilidades relacionales y conciencia social.

Cohen y Sandy (2003) desarrollan una revisión conceptual de las emociones, advirtiendo sobre las tensiones teóricas que generan en los procesos educativos. Estos autores puntualizan su complejidad pedagógica y la influencia que tienen las nociones sobre las/os estudiantes, la enseñanza y el aprendizaje en las prácticas y formas de abordar su desarrollo escolar.

Las definiciones anteriores, entre otras, dan cuenta de distintas posibilidades para enunciar y definir las formas de abordar las emociones en contextos educativos, lo que

\footnotetext{
${ }^{5}$ Collaborative for Academic, Social and Emotional Learning (CASEL) es una organización internacional de académicos/as e investigadores/as dedicados/as al aprendizaje socioemocional. Entre sus colaboradores destacan Daniel Goleman y Roger Weissberg, entre otros/as.
} 
pone de manifiesto que las emociones, la educación y el aprendizaje socioemocional no son conceptos unívocos.

Sin perjuicio de esta amplitud y diversidad conceptual, existen experiencias en Chile acerca del enfoque socioemocional sobre los procesos de aprendizaje. Milicic et al. (2013) implementaron un programa con estudiantes de quinto y sexto año de educación básica, desde el cual rescataron la importancia de formar a los/as docentes respecto a este enfoque. Del mismo modo, se ha detectado la importancia de las relaciones interpersonales para el bienestar socioemocional, como factor que permite construir ambientes afectivos dentro de la escuela (Toro y Berger, 2012).

A pesar de la importancia atribuida a las emociones dentro del ámbito educativo y la urgencia de incluirlas dentro de la formación inicial y continua del profesorado, poco se ha discutido sobre cómo implementar la educación socioemocional en el contexto educativo chileno. Esto convive con la diversidad de aproximaciones conceptuales que hay en esta materia, lo que plantea una pregunta que requiere ser discutida: ¿qué elementos deben ser considerados para la implementación del aprendizaje socioemocional en el contexto educativo chileno? Esta pregunta surge a partir de la revisión de literatura sobre el tema y la especial consideración de algunos antecedentes relevantes sobre nuestro sistema escolar. Entre ellos se incluye la escasa importancia que se le ha dado a la dimensión emocional dentro de los programas educativos chilenos, cuya orientación ha sido criticada por su fuerte énfasis en el desarrollo de habilidades académicas y procedimentales.

Para responder a la pregunta enunciada, el presente ensayo tiene por objetivo discutir aspectos relacionados con el aprendizaje y desarrollo socioemocional que resultan relevantes para implementar y apropiarse de la educación socioemocional como modelo teórico y orientación práctica. Para ello, se recurre a algunas fuentes bibliográficas que serán utilizadas para levantar discusiones y argumentos en torno a la pregunta planteada. Dentro de este trabajo se abordarán dos aspectos de la educación y aprendizaje socioemocional relevantes por sus implicancias psicológicas y educativas. Por un lado, se discutirán algunas consideraciones sobre las emociones como proceso psicológico inserto en las trayectorias de aprendizaje; por otro, se realizará una aproximación a la complejidad que las comunidades educativas -y en especial, el profesorado- deben 
enfrentar, si quieren plantearse un acercamiento educativo que considere las dimensiones sociales y emocionales como componentes claves de sus prácticas.

Esta discusión se instala con la finalidad de identificar consideraciones pertinentes para construir futuras líneas de investigación o intervención en el ámbito educativo sobre esta materia. Además, intenta responder a la prevalencia que el aprendizaje socioemocional ha adquirido en la discusión pública en el contexto reciente, considerando que puede tener interpretaciones variadas.

\section{Hacia una mirada educativa de las emociones}

Diversos autores ponen de relieve el lugar que las emociones tienen en el desarrollo humano y, particularmente, en los procesos de aprendizaje. Al respecto, discuten cómo esta dimensión puede ser entendida y definida, como Delors (1996), quien propone cuatro pilares para la educación del siglo XXI: aprender a conocer, aprender a hacer, aprender a vivir juntos y aprender a ser, destacando este último ámbito. Esta propuesta convive con las miradas de Maturana (1992), quien señala el carácter inevitable de la dimensión emocional en las interacciones y decisiones educativas, y de Casassus (2007), quien rescata la experiencia emocional humana, discutiendo su alejamiento tradicional de los procesos formativos. Estos referentes, entre otros posibles, nos llevan a complejizar la forma en que entendemos el proceso educativo, en tanto conjunto de interacciones sociales que inevitablemente implican procesos emocionales en su seno.

En este contexto, algunas miradas sobre las emociones han considerado que estas tienen igual importancia, al momento de comprender el aprendizaje y desarrollo humano, que los procesos y habilidades cognitivas (García, 2012). Esto implica que, lejos de comprender cognición y emoción como procesos separados, es necesario hacer visible su inevitable interrelación. Para efectos del fenómeno educativo, la emocionalidad se haría presente en las interacciones educativas e influiría en las decisiones que toman los/as estudiantes de forma cotidiana (Ibáñez, 2002), con lo cual su relevancia se hace aun más evidente. 
Considerando lo anterior, proponemos reconocer la importancia de discutir las miradas y concepciones sobre la dimensión emocional de la experiencia humana, al momento de pensar en el aprendizaje social y emocional. Si bien autores como Cohen y Sandy (2003) han relevado la importancia de problematizar concepciones sobre la enseñanza y el aprendizaje, igualmente importante parece ser identificar ciertas miradas acerca de la emoción como parte del desarrollo humano.

Sobre este aspecto, Weinstein (2018) distingue dos perspectivas en torno a las emociones en el contexto educativo. La primera se ha enfocado en entender las emociones como un conjunto de características individuales, que modulan las posibilidades que los sujetos tienen para hacer frente a momentos y circunstancias vitales. La segunda perspectiva corresponde a la mirada social que considera que los factores contextuales resultan determinantes para la experiencia emocional (Zembylas, 2004). Estas dos perspectivas conviven con una tercera, la cual concibe a las emociones como un conjunto de competencias a desarrollar (Casassus, 2007), que adquieren relevancia toda vez que se piense el aprendizaje socioemocional como el desarrollo de dichas competencias.

Como se puede apreciar, la pregunta sobre la naturaleza de las emociones, dentro de la educación socioemocional, no tiene respuestas únicas ni ajenas a un debate epistemológico e incluso político. Dada la amplitud de miradas sobre este fenómeno, y considerando el acuerdo que hay sobre su relevancia para el aprendizaje y desarrollo, se abren preguntas sobre su relevancia para la política pública. Dichas preguntas se relacionan con el lugar que se atribuye a las emociones dentro del proceso de aprendizaje, el cual podría incidir en las formas en que su desarrollo es promovido. Reconociendo que son una dimensión inevitable y constitutiva de la experiencia educativa, la pregunta sobre cómo comprender las emociones no es inocua para los procesos de aprendizaje y desarrollo. Por el contrario, sostenemos la necesidad de reflexionar sobre las creencias y concepciones que los agentes educativos tienen sobre el proceso emocional, dada la relevancia que estas podrían tener en las decisiones cotidianas sobre su abordaje. De esta forma, es necesario discutir aquellas perspectivas que entienden las emociones como fenómeno individual y que podrían llevar a pensar en abordajes limitados a la acción terapéutica, con miras a la reparación de daños o al aprendizaje individual para el manejo de estas. 
Lo anterior nos deja con dos perspectivas que parecen debatirse en el contexto actual, sobre todo a raíz de los desafíos que la sociedad ha vivido a consecuencia de la crisis sanitaria y política. ¿Podría desconocerse hoy la importancia que el contexto social y cultural tiene en la experiencia emocional individual y colectiva? Los cambios en la vida cotidiana, así como en las condiciones económicas y sociales en las que se desarrollan los/as estudiantes chilenos/as, parecen indicar que no. Por el contrario, el carácter excepcional de la situación actual ha evidenciado que las emociones son moduladas por los momentos personales, pero también por las condiciones y procesos que experimentan las sociedades.

El contraste con esta mirada se encuentra en aquellas que aluden a las competencias socioemocionales, donde se asume la emoción como parte de una serie de habilidades que pueden desarrollarse y optimizarse, añadiendo con ello nuevos desafíos a los procesos pedagógicos. Este enfoque es sostenido por iniciativas como las de CASEL que, si bien incluyen una mirada sistémica sobre las formas en que las emociones pueden aprenderse y desarrollarse dentro del contexto educativo, no escapan de algunos dilemas propios de la instalación de estos modelos en el contexto escolar. Por un lado, la instalación de estas miradas revive la discusión sobre la legitimidad de la emoción como ámbito del desarrollo humano y no solo como instrumento para mejorar el rendimiento académico (Pekrun, 2005 en Rebollo et al., 2008). Por otro, conlleva leer conjuntamente el desafío de la competencia socioemocional, a la vez que se le entiende como un fenómeno inevitablemente contextual y cultural. A partir de lo anterior, ¿cómo construir una mirada sobre las emociones con un foco educativo, no patologizante ni reducido al desarrollo de competencias individuales?

Otra arista consiste en desmitificar las emociones, evitando reducirlas a conceptos abstractos o a experiencias negativas o positivas. Entendiendo la importancia que el pensamiento racional ha tenido para comprender otros aspectos de la vida social, es necesario reconocer que la clasificación y organización de la experiencia emocional en categorías estancas ha sido una tendencia que no permite reconocer el valor de cada emoción que suscita el contexto actual. Al respecto, es necesario reconocer que las emociones han acompañado nuestra sobrevivencia y evolución cultural (Equipo Cuidemnos, 2011; Zúñiga y Ligioiz, 2018). De aquí que la existencia de categorías como 
"emociones positivas" o "emociones negativas" responde a la necesidad que tenemos de entender la complejidad de la experiencia, lo que acarrea el riesgo de invisibilizar las bondades que la experiencia emocional comporta para la relación entre el sujeto y su entorno. Se sostiene, a la vez, que esta relación nutre los procesos de aprendizaje y desarrollo, toda vez que permite entender cómo las personas comprenden los cambios que experimentan en sus contextos culturales.

La posibilidad de responder a la complejidad de la experiencia educativa requiere abrir la pregunta sobre las emociones y, con ello, las posibles respuestas que puede ofrecer la institucionalidad educativa ante la experiencia que viven los/as estudiantes y las familias en la actualidad. Sin perjuicio de ello, la pregunta sobre las emociones no es ajena a las preguntas por el sujeto, sus experiencias y las acciones de desarrollo socioemocional.

\section{Desarrollo socioemocional en docentes y estudiantes}

Para "revalorizar el papel de las emociones en nuestra cultura escolar, la formación de profesores es un ámbito prioritario" (Ibáñez, 2002, p.5) y una responsabilidad ineludible para la educación superior (Vivas, 2003), como espacio de formación inicial docente. Sin embargo, se ha evidenciado que, a pesar de la centralidad de la figura de el/la profesor/a, no existe información ni claridad respecto de su rol como promotor/a de la dimensión socioemocional ni tampoco directrices sobre los alcances y límites de este rol, por lo que quienes son profesores/as con jefaturas de curso deben construir su rol desde sus propias experiencias (Toro y Berger, 2012).

El profesorado puede enseñar lo que sabe y lo que considera necesario de enseñar desde su lugar de autonomía y saber profesional. Sin embargo, la pregunta sobre aquellos fenómenos que acompañan el contenido disciplinar demanda fortalecer los procesos de aprendizaje profesional continuo. Por ello es importante abordar el aprendizaje y la educación socioemocional como enfoques presentes desde la formación docente, como un elemento de relevancia en su saber profesional. No obstante, con una educación emocional en fase de sensibilización, quien desarrolla la labor docente se sentirá motivado/a a formarse en esta materia solo por medio de la toma de conciencia sobre la 
importancia y necesidad de la educación emocional, considerando que es una necesidad transversal (Bisquerra, 2020).

Debido a las características de la labor docente, como trabajo de cuidado y práctica relacional, con la responsabilidad de "facilitar el desarrollo de su alumnado en todas las dimensiones de su personalidad" (Vázquez y Escámez, 2010, p.3), es probablemente uno de los sectores profesionales que más precisa de competencias socioemocionales (Bisquerra, 2020). Más aún, debemos considerar la docencia como una praxis que tiene un impacto directo en las/os nuevas/os ciudadanas/os y sus aprendizajes esperados, los cuales convocan no solo conocimientos disciplinares, sino que habilidades que favorezcan la relación entre las personas que conviven en espacios comunes.

Entonces, la figura de el/la docente es crucial para promover un desarrollo socioemocional profundo y significativo en los/as estudiantes, siendo importantes tanto las competencias socioemocionales docentes como sus creencias sobre este tipo de aprendizaje (Schonert-Reichl, 2017). Por ello, resulta relevante brindar espacios reflexivos en torno a los procesos emocionales que convocan a una praxis que considere el desarrollo personal, profesional y ciudadano. Asimismo, se hace necesario reflexionar en torno al rol de influencia de los/as docentes, en tanto modelos y mediadores/as de las experiencias emocionales que se desarrollan en el aula.

En el caso de las/los estudiantes en edad escolar, debemos referirnos a niños, niñas y adolescentes desde un enfoque de derecho, reconociendo -entre otros aspectos- su derecho a participar en las decisiones que les afectan directamente. Lo anterior les ofrece una oportunidad para conocerse a sí mismos/as en diversos contextos y explorarse como seres sociales, emocionales y ciudadanos. A través de la participación en los entornos en los que se desenvuelven, pueden mostrar su presente, construir su futuro y contribuir a las comunidades con las que se vinculan (Unicef, 2010).

Así, se invita a realizar una mirada sobre niños, niñas y adolescentes considerando su capacidad de agencia, desde un enfoque centrado en las fortalezas y no en las limitaciones o ausencias (Balaguer, 2016). Este enfoque comprende a los seres humanos como el resultado de la interacción entre las diversas dimensiones que lo construyen (biológica, psicológica, ecológica e histórica), sosteniendo la plasticidad del desarrollo y la constante 
oportunidad de interactuar con los recursos del sistema. En la misma línea, se invita a una práctica docente que reconozca la importancia de la experiencia emocional en el aprendizaje y en lo relacional, concibiendo estos ámbitos como inseparables dentro de la experiencia de las y los estudiantes, sabiendo que la experiencia emocional contará con diversas características en las distintas personas.

Ahora bien, estudiantes y docentes se encuentran en el aula cotidianamente y su relación es un elemento a analizar en el marco de las emociones que emergen en cada uno/a de ellos/as y en su diálogo cotidiano. Albornoz y Cornejo (2017) plantean que las percepciones que emergen desde los/as docentes hacia sus estudiantes refieren a experiencias que aparecen con manifestaciones diversas. Destacan también la dificultad para abordar el vínculo y las sensaciones contradictorias que surgen en la relación, pudiendo ser a la vez una guerrilla con los/as estudiantes y una fuerza que da sentido y moviliza a seguir educando. La intensidad de esta relación, sin duda, es desafiante para la práctica pedagógica habitual, ya que los/as profesores/as son representantes de una institución escolar y, a la vez, comparten relaciones particulares con cada una/o de sus estudiantes. Otra línea de estudios apunta justamente a la generación de vínculos positivos en la relación de profesor/a jefe/a y estudiante, de acuerdo a los planteamientos de autores como Winnicott respecto a las relaciones tempranas con las figuras de cuidado y Bowlby sobre la teoría del apego (Mena et al., 2015).

En general, abordar la relación entre profesor/a y estudiante requiere entender la importancia de las interacciones, la reciprocidad y la capacidad que tienen de influenciarse mutuamente (González, 2004 en Gordillo et al., 2014), considerando el rol que tienen el contexto y los procesos sociales para promover o dificultar estos vínculos interpersonales. En cualquier caso, la consideración de las subjetividades involucradas en el proceso pedagógico, así como la comprensión de los contextos en los que surge dicha interacción, resultan cruciales toda vez que la implementación de programas o acciones de aprendizaje socioemocional requieren de miradas complejas y sistémicas. Esto es especialmente cierto en el contexto escolar chileno y, en particular, en las comunidades que han vivido las consecuencias sociales y educativas de la crisis sanitaria reciente. 


\section{Hacia una mirada crítica del desarrollo socioemocional en el contexto educativo chileno}

Las discusiones anteriores se han referido a la naturaleza de las emociones, así como a las consideraciones que merecen los agentes y contextos educativos al momento de pensar en la adopción del aprendizaje socioemocional como modelo orientador para la acción educativa. Esto parece particularmente importante y abre la discusión a un tercer elemento, relacionado con el posicionamiento que requiere la discusión sobre este modelo como forma de pensar y ejecutar los procesos de aprendizaje y desarrollo en el contexto actual.

La escuela no se limita a una infraestructura, sino que es un espacio que cobija en sus aulas a personas que, en sus múltiples roles, se desarrollan en torno al aprendizaje. Este último, a su vez, como hemos mencionado anteriormente, no se limita a un currículum que describe contenidos, sino que incluye experiencias que involucran emociones propias ante lo que ocurre en ese espacio y ante las personas con quienes se comparten las aulas.

De esta forma, comprendemos la escuela desde una visión compleja que la concibe como un tejido de componentes heterogéneos inseparablemente asociados y que conforman un todo, desde la paradoja de lo uno y lo múltiple, el orden y el desorden, los azares y las determinaciones. De igual manera, se constituye desde una visión sistémica (Morin, 1995; 1999), en la que hay constante influencia de los sistemas que participan a su alrededor, siendo permeable a los hechos sociales de las comunidades en las que se encuentra inserta.

Es por lo anterior que no podemos ver la formación emocional desde la simplicidad, sino que debemos incorporar en la práctica y en la mirada de proceso los múltiples componentes que toman parte. Lo anterior se enriquece al trabajar en la escuela desde una visión compleja del proceso de aprendizaje, que considera los múltiples factores que lo constituyen y los variados resultados que emergen, y no desde la parcialidad de oficinas o programas.

En esta labor, se visibiliza la tensión entre la necesidad de flexibilizar el currículum y los procesos evaluativos, mientras las labores educativas se desarrollan en un contexto de recurrente rendición de cuentas con carácter punitivo, debido a las altas consecuencias 
que los resultados alcanzados traen a las comunidades escolares (Ascorra et al., 2019). Ante ello, favorecer la flexibilización genera un escenario pertinente para el desarrollo del aprendizaje en un contexto de emergencia. De igual manera, ante la concepción del desarrollo emocional como proceso, hay que tener presente la inviabilidad de estandarizar procesos tan diversos dentro del aula.

Entonces, cabe preguntarse por las condiciones necesarias para promover el aprendizaje socioemocional dentro del sistema educativo. Como propuesta, y en continuidad a lo señalado antes, se plantea visibilizar la escuela desde una visión compleja y sistémica, en la que se identifiquen las características de los entornos en los que participa la comunidad educativa. Cada escuela tiene una realidad propia que se debe conocer como base al momento de pensar en una planificación y en las dimensiones de la convivencia cotidiana.

En la misma línea, se propone una visión del desarrollo emocional que no se limite a aspectos individuales, sino que se complemente y fortalezca desde la dimensión comunitaria que propone la escuela, sobre todo al momento de convivir con una diversidad de construcciones de mundo, las cuales favorecen también el desarrollo de habilidades sociales como la empatía, la asertividad y el respeto. Para ello, estrategias como asambleas, comunidades de curso y estrategias de trabajo colaborativo favorecen el abordaje, en la dinámica relacional, de aspectos emocionales que se vivencian en el aula, así como una resolución de conflictos en base a la construcción conjunta de soluciones.

\section{Reflexiones finales}

Las ideas contenidas en este ensayo tienen como punto de partida el reconocimiento de la importancia del aprendizaje socioemocional en el contexto actual y recorren algunos elementos que parecen relevantes para su implementación en el contexto educativo chileno. Esto es de particular importancia en el escenario actual, donde las comunidades educativas resienten las consecuencias que la crisis sanitaria, social y política ha generado. 
Para ello, se reconoce la importancia de las emociones en los procesos de desarrollo y aprendizaje, destacando su relevancia dentro del contexto social actual, así como su lugar dentro de las interacciones educativas. Este reconocimiento no es solo una constatación, sino además una invitación a promover la reflexión profesional sobre la naturaleza de las emociones y las decisiones que se toman cotidianamente para abordarlas dentro de las prácticas pedagógicas. Dicha reflexión no se refiere exclusivamente a las emociones en tanto concepto abstracto, sino que además requiere considerar a los sujetos que experimentan el aprendizaje socioemocional, rescatando el saber profesional y autónomo de los/as docentes, así como la legitimidad de la experiencia estudiantil. Al considerar estos elementos, se abre el llamado a tener una lectura crítica sobre el aprendizaje socioemocional que no reduzca las emociones a un conjunto de competencias que requieren desarrollarse en lo individual, sino que plantee una transformación profunda de las prácticas y culturas escolares; una lectura que no se limite a una revisión de instrumentos para tener mejores resultados, sino que considere las emociones dentro de la praxis, como constitutivas de la experiencia educativa y humana.

Avanzar en estos desafíos conlleva transformar las lógicas según las cuales los sistemas educativos se rigen, legitimando la necesidad de nuevas prioridades educativas que estén por sobre los resultados de evaluaciones estandarizadas con altas consecuencias. Esto es coherente con una mirada compleja y sistémica de la escuela que nos invita a analizar su relación con el sistema social en el que se inscribe. Al realizar lo anterior, se podrá evitar la reproducción de lógicas que instrumentalizan las emociones para la mejora de resultados académicos, así como dinámicas de segregación y patologización estudiantil dentro de las comunidades escolares. Ello será posible cuando la pregunta sobre el aprendizaje socioemocional se comprenda como un desafío que no solo es contingente en contextos de emergencia, ya que también debe situarse de manera permanente en el quehacer específico del sistema educativo chileno. De ese modo será posible responder de manera situada a los desafíos globales que se nos plantean en estos días. 


\section{Referencias}

Agencia de Calidad de la Educación. (2018). La importancia de las creencias y emociones en el desarrollo personal y académico de los estudiantes. Recuperado de http://archivos.agenciaeducacion.cl/policy_brief_Final_N3.pdf

Albornoz, N. y Cornejo, R. (2017). Discursos docentes sobre el vínculo con sus estudiantes: tensiones, enfrentamiento y distancia. Estudios Pedagógicos, 43(2), $7-25$.

Ascorra, P., Carrasco, C., López, V. y Morales, M. (2019). Políticas de convivencia escolar en tiempos de rendición de cuentas. Archivos Analíticos de Políticas Educativas, 27(31). http://dx.doi.org/10.14507/epaa.27.3526

Balaguer, A. (2016). Desarrollo positivo adolescente desde la perspectiva personal. Relación con variables sociodemográficas, contextuales y rendimiento académico. Dialnet Plus. https://dialnet.unirioja.es/servlet/tesis?codigo=137670

Bisquerra, R. (2000). Educación emocional y bienestar. Wolters Kluwer.

Casassus, J. (2007). La educación del ser emocional. Indigo - Cuarto Propio.

Collaborative for Academic, Social, and Emotional Learning. (2017). Competencias del aprendizaje social y emocional (SEL). https://casel.org/wpcontent/uploads/2019/12/CASEL-wheel-competencies-Spanish.pdf

Cohen, J. y Sandy, S. (2003). Perspectives in Social-Emotional Education: Theoretical Foundations and New Evidence-Based Developments in Current Practice. Perspectives in Education, 21(4), 41-54.

Cornejo, R. (2012). Nuevos sentidos del trabajo docente: Un análisis psicosocial del bienestar/malestar, las condiciones de trabajo y las subjetividades de los/as docentes en el Chile neoliberal (t esis doctoral). Universidad de Chile, Chile.

Delors, J. (1996). La educación encierra un tesoro. Informe a la UNESCO de la Comisión Internacional sobre la educación para el siglo XXI. Santillana - Ediciones Unesco. 
Equipo Cuidem-nos (2011). El bienestar del docente. Vivir bien educando: estrategias para conseguir satisfacción profesional y personal. Graó.

García, J. (2012). La educación emocional, su importancia en el proceso de aprendizaje. Revista de Educación, $\quad 36(1), \quad$ 1-24. https://www.redalyc.org/articulo.oa?id=44023984007

Gordillo, M., Ruiz, M., Sánchez, S. y Calzado, Z. (2014). Clima afectivo en el aula: vínculo emocional maestro-alumno. Psicología, ciencia y profesión: afrontando la realidad, sistemas, cibernética e informática, 11(2), 195-201.

Ibáñez, N. (2002). Las emociones en el aula. Estudios Pedagógicos, (28), 31-45.

Maturana, H. (1992). Emociones y lenguaje en educación y política. Ediciones Pedagógicas Chilenas.

Mena, I., Bugueño, X. y Valdés, A. (2015). Vínculo pedagógico positivo: principios para su desarrollo. http://valoras.uc.cl/images/centrorecursos/docentes/RolDocente/Fichas/Vinculo-pedagogico-positivo.pdf

Milicic, N., Alcalay, L., Berger, Ch. y Álamos, P. (2013). Aprendizaje socioemocional en estudiantes de quinto y sexto grado: presentación y evaluación de impacto del programa BASE. Ensaio: Avaliação e Políticas Públicas em Educação, 21(81), 645-666.

Mesa Social Covid (2020). Salud mental en situación de pandemia. Gobierno de Chile. https://s3.amazonaws.com/gobcl-prod/public_files/Campañas/MesaCovid19/documentos/salud-mental.pdf

Ministerio de Salud (2020). Guía práctica de bienestar emocional: Cuarentena en tiempos de COVID19. Mesa Técnica de Salud Mental en la Gestión de Riesgos de Desastres - MINSAL https://cdn.digital.gob.cl/public_files/Campañas/CoronaVirus/documentos/2020.04.12_GUIA-PRACTICA-CUARENTENA-ENTIEMPOS-DE-COVID19_final.pdf 
Ministerio de Educación (2020). Plan Nacional Convivencia Escolar y Aprendizaje Socioemocional. $\quad$.http://convivenciaescolar.mineduc.cl/plan-nacionalconvivencia-escolar-y-aprendizaje-socioemocional/.

Morin, E. (1995). Introducción al pensamiento complejo. Gedisa.

Morin, E. (1999). Los siete saberes necesarios para la educación del futuro. Unesco.

Organización para la Cooperación y el Desarrollo Económicos. (2020). Schooling Disrupted, Schooling Rethought. How the COVID-19 Pandemic is Changing Education.https://globaled.gse.harvard.edu/files/geii/files/education_continuity_ v3.pdf

Rebollo, M., García, R., Barragán, R., Buzón, O. y Vega, L. (2008). Las emociones en el $\begin{array}{llll}\text { aprendizaje } & \text { online. } & \text { RELIEVE, } & 14(1),\end{array}$ http://www.uv.es/RELIEVE/v14n1/RELIEVEv14n1_2.htm.

Schonert-Reichl, K. (2017). Social and Emotional Learning and Teachers. The Future of Children, 27(1), 137-155.

Toro, L. y Berger, Ch. (2012). Percepción de profesores jefe de quinto y sexto básico de su rol en el aprendizaje socioemocional de los estudiantes. Diálogos Educativos, 23(12), 16-37. http://www.dialogoseducativos.cl/revistas/n23/toro

Fondo de las Naciones Unidas para la Infancia. (2010). Desarrollo adolescente y derechos humanos. https://www.unicef.org/venezuela/sites/unicef.org.venezuela/files/201902/Desarrollo\%20Adolescente\%20y\%20DDHH.pdf

Fondo de las Naciones Unidas para la Infancia. (2020). Guidance on Distance Learning Modalities. To Reach All Children and Youth During School Closures. https://www.unicef.org/rosa/reports/guidance-distance-learning-modalitiesreach-all-children-and-youth-during-school-closures

Vázquez, V., y Escámez, J. (2010). La profesión docente y la ética del cuidado. Revista electrónica de investigación educativa, (12), 1-17. 
http://www.scielo.org.mx/scielo.php?script=sci_arttext\&pid=S 1607$40412010000300001 \& \operatorname{lng}=\mathrm{es} \& \operatorname{tlng}=\mathrm{es}$.

Vivas, M. (2003). La educación emocional: conceptos fundamentales. Sapiens, Revista Universitaria de Investigación, 4(2).

Weinstein, E. (2018). Características emocionales del trabajo de docentes de colegio: un estudio cualitativo a partir de incidentes críticos en el aula (tesis de magíster). Universidad de Chile, Chile.

Zembylas, M. (2004). The Emotional Characteristics of Teaching: An Ethnographic Study of One Teacher. Teaching and Teacher Education, (20), 185-201.

Zuñiga, H. y Ligioiz, M. (2018). Neurocomunicación. El gran salto de las comunicaciones humanas. Rigden Institut Gestalt. 